\title{
Measuring and making decisions for social reciprocity
}

\author{
Antonio Solanas and DaVID LeIVa \\ University of Barcelona, Barcelona, Spain \\ Vicenta SiERra \\ Ramon Llull University, Barcelona, Spain \\ AND \\ Lluís SALAFranca \\ University of Barcelona, Barcelona, Spain
}

\begin{abstract}
Social reciprocity may explain certain emerging psychological processes likely to be founded on dyadic relations. Although indexes and statistics have been proposed to measure and make statistical decisions regarding social reciprocity in groups, these tools were generally developed to identify association patterns rather than to quantify the discrepancies between what each individual addresses to his or her partners and what is received from those partners in return. Additionally, social researchers' interest extends beyond measuring groups at the global level because dyadic and individual measurements are also necessary for proper descriptions of social interactions. This study is concerned with a new statistic for measuring social reciprocity at the global level and with decomposing that statistic in order to identify which dyads and individuals account for a significant part of asymmetry in social interactions. In addition to a set of indexes, some exact analytical results are derived, and a way of making statistical decisions is proposed.
\end{abstract}

Social psychology research has been focused mainly on the individualistic approach, even though social phenomena involve two or more individuals. Perhaps the most important drawback of the individualistic approach is that it ignores the social context within which individuals are embedded. This probably explains why dyadic analysis has increasingly been applied to measure and analyze groups (Kenny, 1994; Kenny, Kashy, \& Cook, 2006). Furthermore, dyadic analysis enables social researchers to determine interaction effects between individuals in a dyad - a main effect that cannot be known by using the individualistic approach, since individualistic research methods cannot reveal patterns of mutual influence and interdependence (Bond, Horn, \& Kenny, 1997; Campbell \& Kashy, 2002; Gonzalez \& Griffin, 1999; Griffin \& Gonzalez, 1995).

Asymmetric social relationships in pairs of individuals are not uncommon. A simple example is the number of times individuals help each other. Square matrices are useful for representing this and other similar examples of asymmetric relationships, where rows and columns correspond, respectively, to a set of individuals in one mode and to the same individuals in a different mode. In general, rows represent individuals as actors or initiators of some kind of behavior; columns correspond to them as partners or receivers of actors' behaviors. These sociomatrices are usually asymmetrical, and this lack of symmetry renders some established statistical methods inappropriate. However, since asymmetry embodies some important information contained in the data, asymmetric data should be analyzed (Saito \& Yadohisa, 2005). Hence, although one could carry out mathematical transformations and thus obtain symmetric matrices, this would ignore departures from symmetry that may be informative.

A number of studies have already dealt with asymmetrical sociomatrices. Regarding dominance hierarchy, two related linearity indexes have been developed to measure this attribute in many groups, and statistical methods were also proposed to test whether the linearity is stronger than that expected by chance (Kendall \& Babington Smith, 1940; Landau, 1951). More recent research has proposed an improved method for those cases in which measurements include tied and/or unknown relationships (de Vries, 1995). Other statistical methods have been developed to rank individuals as a function of the outcomes of dyadic dominance encounters (de Vries, 1998; de Vries \& Appleby, 2000; de Vries, Stevens, \& Vervaecke, 2006). As for reciprocity, interchange, and other social interaction patterns, some statistical methods consisting of computing the association between two matrices have been recommended for analyzing interaction data, and, in order to avoid distorting effects due to mutual dependency, permutation tests should be applied (Hemelrijk, 1990a, 1990b).

A. Solanas, antonio.solanas@ub.edu 
In the context of social psychology, any sociomatrix can be decomposed into its variance components (Warner, Kenny, \& Stoto, 1979). For instance, the social relations model (SRM; Kenny \& La Voie, 1984) is based on roundrobin designs that allow one to gather interaction data. Actor, partner, and relationship effects can be estimated, and a random effects ANOVA is used to partition variance into its components. This statistical model also enables social researchers to estimate dyadic and generalized reciprocity by means of correlation coefficient values, although it does not allow social reciprocity to be measured at the global level.

In general, the statistical methods mentioned above use association indexes to estimate global reciprocity in social systems. Although these indexes undoubtedly enable social researchers to identify the specific social relations that emerge in social interactions, association indexes do not measure the correspondence between what an individual gives others and what is received in return. The $d i-$ rectional consistency index (van Hooff \& Wensing, 1987), which is based on absolute differences between what each member of a pair gives to the other and what is received from the other, enables researchers to obtain a measure of global reciprocity, in which the magnitude of the behavior is taken into account. Recently, another statistic for measuring global reciprocity has been developed to quantify the discrepancy between what is addressed to others and what is received in return (Solanas, Salafranca, Riba, Sierra, \& Leiva, 2006). This reciprocity statistic can be partitioned in such a way that people who contribute more to the lack of reciprocity can be identified; dyadic and generalized reciprocity may also be measured. This reciprocity statistic, called the skew-symmetry statistic, can also be tested for statistical significance (Leiva, Solanas, \& Salafranca, 2008). The skew-symmetry statistic ranges from 0 to .5 , and the random variable is located at the denominator. Hence, it would be better to develop a statistic whose values range from 0 to 1 and, more important, one for which exact analytical results can be obtained. Furthermore, in order to make comparisons between different studies, the reciprocity statistic should be normalized for minimum and maximum values. Regarding statistical inference, there is also a need for a statistical method capable of making statistical decisions.

The main aim of the present study is to propose a new statistic for quantifying social reciprocity and to propose a corresponding statistical method for making decisions regarding social reciprocity in groups. Although we assume that social phenomena depend on dyadic interactions and, hence, that the statistic should be based on dyadic data, we propose new indexes of social reciprocity at global, dyadic, and individual levels. Our study could be useful for those researchers who are interested in family interactions, play relationships, cooperative learning, agonistic behaviors, and other topics.

\section{A New Index for Measuring Social Reciprocity}

Consider $n$ individuals, who are labeled $i=1,2, \ldots, n$ in an experiment involving dyadic interactions. Let $c_{i j}=$ $c_{j i}$ be the number of observed interactions between the individuals $i$ and $j$, and let $x_{i j}$ be the number of times $i$ is recorded to address a specific behavior to $j$. Note that $c_{i j}=x_{i j}+x_{j i}$, since $x_{i j}$ and $x_{j i}$, respectively, represent the number of behaviors each individual of the dyad addresses to the other. It is assumed that the probability that an individual $i$ addresses behavior to $j$ in each single interaction remains steady during the period of time the observations are made. The parameter $\pi_{i j}$ denotes the probability that individual $i$ addresses the behavior of interest to $j$. It should be noted that $\pi_{i j}+\pi_{i i}=1$, since it is assumed that only one individual of each dyad addresses the behavior to the other in each social interaction. Thus, social reciprocity in a group can be represented in a matrix $\Pi$, as follows:

$$
\Pi=\left(\begin{array}{ccccc}
0 & \pi_{12} & \pi_{13} & \cdots & \pi_{1 n} \\
1-\pi_{12} & 0 & \pi_{23} & \cdots & \pi_{2 n} \\
1-\pi_{13} & 1-\pi_{23} & 0 & \cdots & \vdots \\
\vdots & \vdots & \vdots & \ddots & \vdots \\
1-\pi_{1 n} & 1-\pi_{2 n} & \cdots & \cdots & 0
\end{array}\right) .
$$

The parameters of the matrix $\Pi$ can be used to define a new measurement of social reciprocity, since these parameters contain the essential information for quantifying dyadic reciprocity among all pairs of individuals. Note that the values of $\pi_{i j}$ are unknown, since they are parameters; note also that social reciprocity can be described by means of $n(n-1) / 2$ independent parameters. It should be stressed that independence among dyads is assumed.

In order to define the new index, the trace of the product matrix $\Pi^{\prime} \Pi$ is obtained as follows:

$$
\begin{aligned}
\operatorname{tr}\left(\boldsymbol{\Pi}^{\prime} \boldsymbol{\Pi}\right) & =\sum_{i=1}^{n} \sum_{\substack{j=1 \\
j \neq i}}^{n} \pi_{i j}^{2}=\sum_{i=1}^{n} \sum_{j=i+1}^{n}\left[\pi_{i j}^{2}+\left(1-\pi_{i j}\right)^{2}\right] \\
& =\frac{n(n-1)}{2}+2 \sum_{i=1}^{n} \sum_{j=i+1}^{n} \pi_{i j}^{2}-2 \sum_{i=1}^{n} \sum_{j=i+1}^{n} \pi_{i j} .
\end{aligned}
$$

Note that $\operatorname{tr}\left(\boldsymbol{\Pi}^{\prime} \boldsymbol{\Pi}\right)$ will take its maximum value if $\pi_{\mathrm{ij}}=0$ or $\pi_{\mathrm{ij}}=1$ for all individuals $i$ and $j$. Thus, the maximum value of $\operatorname{tr}\left(\boldsymbol{\Pi}^{\prime} \boldsymbol{\Pi}\right)$ is equal to

$$
\max \left[\operatorname{tr}\left(\boldsymbol{\Pi}^{\prime} \boldsymbol{\Pi}\right)\right]=\frac{n(n-1)}{2} .
$$

Thus, the maximum value only depends on the number of individuals in a group and corresponds to those cases in which there is a complete lack of reciprocity in every dyad.

The minimum value of $\operatorname{tr}\left(\boldsymbol{\Pi}^{\prime} \boldsymbol{\Pi}\right)$ corresponds to $\pi_{i j}=$ $1 / 2$ for all individuals $i$ and $j$. Thus, we can obtain the minimum value of $\operatorname{tr}\left(\boldsymbol{\Pi}^{\prime} \boldsymbol{\Pi}\right)$ as follows:

$$
\begin{aligned}
\min \left[\operatorname{tr}\left(\boldsymbol{\Pi}^{\prime} \boldsymbol{\Pi}\right)\right]= & \frac{n(n-1)}{2}+2 \frac{n(n-1)}{2}\left(\frac{1}{2}\right)^{2} \\
& -2 \frac{n(n-1)}{2}\left(\frac{1}{2}\right)=\frac{n(n-1)}{4} .
\end{aligned}
$$

The minimum value of $\operatorname{tr}\left(\boldsymbol{\Pi}^{\prime} \boldsymbol{\Pi}\right)$ once again depends only on the number of individuals in a group. 
We can now define a new index to measure overall social reciprocity in groups, taking into account that it should be bounded between 0 and 1 , as follows:

$$
\Phi_{r}=\frac{\operatorname{tr}\left(\boldsymbol{\Pi}^{\prime} \boldsymbol{\Pi}\right)-\min \left[\operatorname{tr}\left(\boldsymbol{\Pi}^{\prime} \boldsymbol{\Pi}\right)\right]}{\max \left[\operatorname{tr}\left(\boldsymbol{\Pi}^{\prime} \boldsymbol{\Pi}\right)\right]-\min \left[\operatorname{tr}\left(\boldsymbol{\Pi}^{\prime} \boldsymbol{\Pi}\right)\right]}, 0 \leq \Phi_{r} \leq 1 .
$$

This index takes its minimum value if all $\pi_{i j}$ are equal to .5 ; the maximum value corresponds to those cases in which $\pi_{i j}=1$ and $\pi_{j i}=0$ or $\pi_{i j}=0$ and $\pi_{j i}=1$ for every dyad. If the index value equals 0 , it indicates that individuals are completely reciprocal, with respect to others. On the other hand, the group will show a large lack of reciprocity if $\Phi_{r}$ is close to 1 . In other words, if $\Phi_{r}=0$, it denotes symmetrical relationships among all individuals regarding the behavior of interest; $\Phi_{r}=1$ corresponds to the maximum level of asymmetry.

It can be shown by means of some algebraic operations that the index $\Phi_{r}$ can also be obtained from the following equation:

$$
\Phi_{r}=\frac{4 t r\left(\boldsymbol{\Pi}^{\prime} \boldsymbol{\Pi}\right)-n(n-1)}{n(n-1)}=\frac{8 \sum_{i=1}^{n} \sum_{j=i+1}^{n} \pi_{i j}^{2}-8 \sum_{i=1}^{n} \sum_{j=i+1}^{n} \pi_{i j}+n(n-1)}{n(n-1)}=1-\frac{8\left(\sum_{i=1}^{n} \sum_{j=i+1}^{n} \pi_{i j}-\sum_{i=1}^{n} \sum_{j=i+1}^{n} \pi_{i j}^{2}\right)}{n(n-1)} .
$$

Note in the last expression that if $\pi_{i j}=\pi$ for all $i<j$, the mathematical expression can be written in the following way:

$$
\Phi_{r}=1-\frac{8\left[\frac{n(n-1)}{2} \pi-\frac{n(n-1)}{2} \pi^{2}\right]}{n(n-1)}=1-4\left(\pi-\pi^{2}\right) .
$$

This means that if $\pi_{i j}=\pi$ for all $i<j$, the value of the index does not depend on the number of individuals but is only a function of $\pi$. Figure 1 shows how the value of the index $\Phi_{r}$ increases as a function of the value $\pi$. As an example, suppose that the matrix $\Pi$ of a group is as follows:

$$
\boldsymbol{\Pi}=\left(\begin{array}{llll}
0 & .8 & .8 & .8 \\
.2 & 0 & .8 & .8 \\
.2 & .2 & 0 & .8 \\
.2 & .2 & .2 & 0
\end{array}\right) .
$$

Then the index $\Phi_{r}$ is equal to

$$
\Phi_{r}=1-4\left(\pi-\pi^{2}\right)=1-4\left(.8-.8^{2}\right)=.36 .
$$

Now we can define the index $\Psi_{r}$ as follows:

$$
\Psi_{r}=1-\Phi_{r}, \quad 0 \leq \Psi_{r} \leq 1
$$

Conversely to $\Phi_{r}$, the index $\Psi_{r}$ takes a value close to 0 if dyadic interactions within a group are asymmetrical; its value is equal to 1 if there is complete reciprocation.

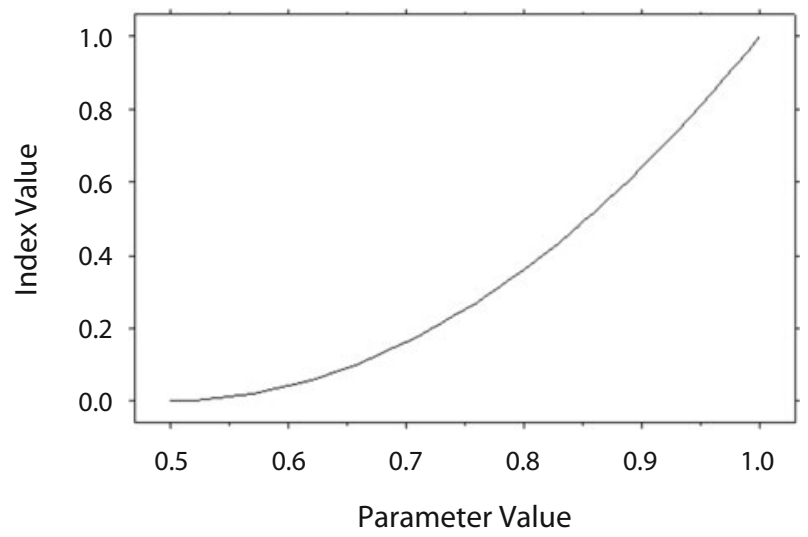

Figure 1. The values of index $\Phi_{r}$ for different parameter values, in which $\pi_{i j}=\pi$ for all $i<j$. 


\section{Estimating Asymmetry in Social Relations}

Because they collect only empirical data, social researchers do not know the value of $\Phi_{r}$. Thus, an estimator of asymmetry in social relations is required for obtaining some information about social reciprocity. An estimator of the index $\Phi_{r}$ can be defined as follows:

$$
\hat{\Phi}_{r}=\frac{\sum_{i=1}^{n} \sum_{\substack{j=1 \\ j \neq i}}^{n} x_{i j}^{2} / c_{i j}^{2}-\min \left(\sum_{i=1}^{n} \sum_{\substack{j=1 \\ j \neq i}}^{n} x_{i j}^{2} / c_{i j}^{2}\right)}{\max \left(\sum_{\substack{i=1 \\ j}}^{n} \sum_{\substack{j=1 \\ j \neq i}}^{n} x_{i j}^{2} / c_{i j}^{2}\right)-\min \left(\sum_{\substack{i=1 \\ j}}^{n} \sum_{j=1}^{n} x_{i j}^{2} / c_{i j}^{2}\right)}, 0 \leq \hat{\Phi}_{r} \leq 1 .
$$

In order to standardize $\hat{\Phi}_{r}$ values, the following maximum value should be obtained:

$$
\max \left(\sum_{\substack{i=1 \\ j}}^{n} \sum_{\substack{j=1 \\ j \neq i}}^{n} x_{i j}^{2} / c_{i j}^{2}\right)=\sum_{i=1}^{n} \sum_{j=i+1}^{n} c_{i j}^{2} / c_{i j}^{2}=n(n-1) / 2 .
$$

Three cases should be distinguished to obtain the minimum value. First, if all $c_{i j}$ are even, the minimum value is obtained from the following expression:

$$
\min \left(\sum_{\substack{i=1 \\ j=1 \\ j \neq i}}^{n} x_{i j}^{2} / c_{i j}^{2}\right)=\sum_{i=1}^{n} \sum_{\substack{j=1 \\ j \neq i}}^{n} \frac{\left(c_{i j} / 2\right)^{2}}{c_{i j}^{2}}=\sum_{i=1}^{n} \sum_{\substack{j=1 \\ j \neq i}}^{n} \frac{c_{i j}^{2}}{4 c_{i j}^{2}}=\frac{n(n-1)}{4} .
$$

Second, if all $c_{i j}$ are odd, the minimum value is equal to

$$
\min \left(\sum_{i=1}^{n} \sum_{\substack{j=1 \\ j \neq i}}^{n} x_{i j}^{2} / c_{i j}^{2}\right)=\sum_{i=1}^{n} \sum_{j=i+1}^{n}\left[\frac{\left(c_{i j}-1\right)^{2}}{4 c_{i j}^{2}}+\frac{\left(c_{i j}+1\right)^{2}}{4 c_{i j}^{2}}\right]=\frac{n(n-1)}{4}+\frac{1}{2} \sum_{i=1}^{n} \sum_{j=i+1}^{n} \frac{1}{c_{i j}^{2}} .
$$

Third, in the general case, if there are $p$ and $n(n-1) / 2-p$ dyads in which the values $c_{i j}$ are even and odd, respectively, the minimum value equals

$$
\min \left(\sum_{\substack { i=1 \\
\begin{subarray}{c}{j=1 \\
j \neq i{ i = 1 \\
\begin{subarray} { c } { j = 1 \\
j \neq i } }\end{subarray}}^{n} x_{i j}^{2} / c_{i j}^{2}\right)=\frac{2 p}{4}+\frac{2\left[\frac{n(n-1)}{2}-p\right]}{4}+\frac{1}{2} \sum_{i=1}^{n} \sum_{\substack{j=i+1 \\
c_{i j}}}^{n} \frac{1}{c_{i j}^{2}}=\frac{n(n-1)}{4}+\frac{1}{2} \sum_{i=1}^{n} \sum_{\substack{j=i+1 \\
c_{i j} \text { odd }}}^{n} \frac{1}{c_{i j}^{2}} .
$$

We denote this minimum value by $m$ in what follows.

We have previously supposed that $c_{i j} \neq 0$ for all $i$ and $j$, where $i \neq j$. If there are dyads for which $c_{i j}=0$, the maximum is as follows:

$$
z=\max \left(\sum_{\substack { i=1 \\
\begin{subarray}{c}{j=1 \\
j \neq i \\
c_{i j} \neq 0{ i = 1 \\
\begin{subarray} { c } { j = 1 \\
j \neq i \\
c _ { i j } \neq 0 } }\end{subarray}}^{n} x_{i j}^{2} / c_{i j}^{2}\right)=\operatorname{card}\left\{c_{i j} \neq 0\right\}
$$

for $i<j$, where card denotes the number of different dyads for which $c_{i j}$ is not equal to 0 . With regard to the minimum,

$$
m=\min \left(\sum_{\substack { i=1 \\
\begin{subarray}{c}{j=1 \\
j \neq i \\
c_{i j} \neq 0{ i = 1 \\
\begin{subarray} { c } { j = 1 \\
j \neq i \\
c _ { i j } \neq 0 } }\end{subarray}}^{n} x_{i j}^{2} / c_{i j}^{2}\right)=\frac{z}{2}+\frac{1}{2} \sum_{\substack{i=1 \\
c_{i j} \text { odd }}}^{n} \frac{1}{c_{i j}^{2}}
$$

Now, the expression corresponding to the statistic $\hat{\Phi}_{r}$ can be written as follows: 


$$
\hat{\Phi}_{r}=\frac{\sum_{i=1}^{n} \sum_{\substack{j=1 \\ j \neq i}}^{n} x_{i j}^{2} / c_{i j}^{2}-m}{n(n-1) / 2-m} .
$$

If there are some $c_{i j}=0$, the statistic equals

$$
\hat{\Phi}_{r}=\frac{\sum_{\substack { i=1 \\
\begin{subarray}{c}{j=1 \\
j \neq i \\
c_{i j} \neq 0{ i = 1 \\
\begin{subarray} { c } { j = 1 \\
j \neq i \\
c _ { i j } \neq 0 } }\end{subarray}}^{n} x_{i j}^{2} / c_{i j}^{2}-m}{z-m} .
$$

\section{Decomposing Individuals' Contributions to Social Asymmetry}

Note that the following expression enables us to know each individual's contribution to asymmetry in social interactions:

$$
\hat{\Phi}_{r}=\frac{\sum_{i=1}^{n} \sum_{\substack{j=1 \\ j \neq i \\ c_{i j} \neq 0}}^{n} x_{i j}^{2} / c_{i j}^{2}-m}{z-m}=\sum_{i=1}^{n} \frac{\sum_{\substack{j=1 \\ j \neq i \\ c_{i j} \neq 0}}^{n}\left(x_{i j}^{2} / c_{i j}^{2}-\frac{1}{4}\right)-\frac{1}{2} \sum_{\substack{j=i+1 \\ c_{i j} \text { odd }}}^{n} \frac{1}{c_{i j}^{2}}}{z-m}=\sum_{i=1}^{n} \hat{\phi}_{i a},
$$

where $\hat{\phi}_{i a}$ denotes the contribution of individual $i$ to asymmetry as an actor. Note that this quantity is a measure at the individual level. Positive values for $\hat{\phi}_{i a}$ denote that individuals address more behavior to others than they receive in return. On the other hand, negative values correspond to those individuals who address less behavior to others than they receive in return. In addition, the following expression provides the contribution of individual $j$ to asymmetry as a partner:

$$
\hat{\Phi}_{r}=\sum_{j=1}^{n} \frac{\sum_{\substack{i=1 \\ i \neq j}}^{n}\left(x_{j i}^{2} / c_{i j}^{2}-\frac{1}{4}\right)-\frac{1}{2} \sum_{\substack{i=j+1 \\ c_{i j} \text { odd }}}^{n} \frac{1}{c_{i j}^{2}}}{z-m}=\sum_{j=1}^{n} \hat{\phi}_{p j} .
$$

A positive value of this measurement means that the individual receives more behavior from others than she or he gives in return; negative values indicate the opposite. Now, note that the expression for $\hat{\Phi}_{r}$ can be written as follows:

$$
\begin{aligned}
& \sum_{\substack{j=1 \\
j \neq i}}^{n}\left(x_{i j}^{2} / c_{i j}^{2}-\frac{1}{4}\right)-\frac{1}{2} \sum_{\substack{j=i+1 \\
c_{i j} \text { odd }}}^{n} \frac{1}{c_{i j}^{2}} \sum_{\substack{j=1 \\
j \neq i \\
j \neq 1}}^{n}\left(4 x_{i j}^{2} / c_{i j}^{2}-1\right)-2 \sum_{\substack{j=i+1 \\
c_{i j} \text { odd }}}^{n} \frac{1}{c_{i j}^{2}} \\
& \hat{\Phi}_{r}=\sum_{i=1}^{n} \frac{c_{\substack{j \neq i \\
c_{i j} \neq 0}}^{c_{i j} \text { odd }} \text { ij }}{z-m}=\sum_{i=1}^{n} \frac{\substack{j=1 \\
j \neq i}}{c_{i j} \neq 0} \\
& \sum_{\substack{j=1 \\
j \neq i}}^{n}\left(4 x_{i j}^{2} / c_{i j}^{2}-1\right)-\sum_{\substack{j=1 \\
j \neq i}}^{n} \frac{1}{c_{i j}^{2}} \\
& =\sum_{i=1}^{n} \frac{\substack{j \neq i \\
c_{i j} \neq 0}}{4 z-4 m}=\sum_{i=1}^{n} \hat{\phi}_{i a}=\sum_{i=1}^{n} \sum_{j=i+1}^{n}\left(\hat{\phi}_{i j}+\hat{\phi}_{j i}\right)=\sum_{i=1}^{n} \sum_{j=i+1}^{n} \hat{\Phi}_{i j} \text {, }
\end{aligned}
$$

where $\hat{\Phi}_{i j}$ corresponds to each dyad's contribution to asymmetry. This measure is a dyadic one, and, in particular, it quantifies the contribution of each dyad to the overall asymmetry in groups. Additionally, $\hat{\phi}_{i j}$ corresponds to a directional dyadic measure. Specifically, it measures the contribution of individual $i$ to asymmetry that is due to individual $j$.

These dyadic and individual contributions to asymmetry can be useful for identifying those individuals and dyads that are associated with larger differences between what is given and what is received from others.

\section{Mathematical Expectancy of $\hat{\boldsymbol{\Phi}}_{\boldsymbol{r}}$}

We denote the expected value of $\hat{\Phi}_{r}$ by $E\left[\hat{\Phi}_{r}\right]$. Note that we can write the expression for $\hat{\Phi}_{r}$ as follows:

$$
\hat{\Phi}_{r}=\frac{2 \sum_{i=1}^{n} \sum_{\substack{j=1 \\ j \neq i}}^{n} x_{i j}^{2} / c_{i j}^{2}-2 m}{n(n-1)-2 m} .
$$


The mathematical expectancy of the estimator under a specific null hypothesis (see Appendix A) is

$$
E\left[\hat{\Phi}_{r}\right]=\frac{2\left[\sum_{i=1}^{n} \sum_{j=i+1}^{n} 1 / c_{i j}+\sum_{i=1}^{n} \sum_{\substack{j=1 \\ j \neq i}}^{n} \frac{\pi_{i j}^{2}\left(c_{i j}-1\right)}{c_{i j}}-m\right]}{n(n-1)-2 m} .
$$

If $\pi_{i j}=.5$ for all $i$ and $j$, the expected value equals

$$
E\left[\hat{\Phi}_{r}\right]=\frac{2 \sum_{i=1}^{n} \sum_{j=i+1}^{n} 1 / c_{i j}+\sum_{i=1}^{n} \sum_{\substack{j=1 \\ j \neq i}}^{n} \frac{c_{i j}-1}{2 c_{i j}}-2 m}{n(n-1)-2 m} .
$$

Furthermore, if $\pi_{i j}=.5$ for each $i$ and $j$ individual and $c_{i j}=c$ for all dyads,

$$
E\left[\hat{\Phi}_{r}\right]=\frac{n(n-1)(c+1)-4 c m}{2 n(n-1) c-4 c m} .
$$

Knowing the expected value for the proposed whole statistic allows social researchers to make proper comparisons and suitable decisions, since the estimator is biased. In other words, if complete reciprocation is supposed, note that the expected value is not equal to 0 .

\section{Standard Error of $\hat{\boldsymbol{\Phi}}_{r}$}

The variance of $\hat{\Phi}_{r}$ can be expressed as follows:

$$
\begin{aligned}
\sigma^{2}\left(\hat{\Phi}_{r}\right) & =E\left[\hat{\Phi}_{r}^{2}\right]-E^{2}\left[\hat{\Phi}_{r}\right]=E\left[\left(\frac{2 \sum_{i=1}^{n} \sum_{j=1}^{n} x_{i j}^{2} / c_{i j}^{2}-2 m}{n(n-1)-2 m}\right)^{2}\right]-E^{2}\left[\hat{\Phi}_{r}\right] \\
& =4[n(n-1)-2 m]^{-2} E\left[\left(\sum_{i=1}^{n} \sum_{j=1}^{n} x_{i j}^{2} / c_{i j}^{2}-m\right)^{2}\right]-E^{2}\left[\hat{\Phi}_{r}\right] .
\end{aligned}
$$

Note that $E^{2}\left[\hat{\Phi}_{r}\right]$ is known because the expected value for the estimator has already been derived. Now the other term of the previous expression is decomposed as follows:

$$
E\left[\left(\sum_{i=1}^{n} \sum_{j=1}^{n} x_{i j}^{2} / c_{i j}^{2}-m\right)^{2}\right]=E\left[\left(\sum_{i=1}^{n} \sum_{j=1}^{n} x_{i j}^{2} / c_{i j}^{2}\right)^{2}\right]-2 m E\left[\sum_{i=1}^{n} \sum_{j=1}^{n} x_{i j}^{2} / c_{i j}^{2}\right]+m^{2},
$$

where

$$
2 E\left[\sum_{i=1}^{n} \sum_{j=1}^{n} x_{i j}^{2} / c_{i j}^{2}\right]=2 \sum_{i=1}^{n} \sum_{j=1}^{n} E\left[x_{i j}^{2}\right] / c_{i j}^{2}=E\left[\hat{\Phi}_{r}\right][n(n-1)-2 m]+2 m .
$$

Then,

$$
\begin{aligned}
\sigma^{2}\left(\hat{\Phi}_{r}\right)= & \frac{4\left(E\left[\left(\sum_{i=1}^{n} \sum_{j=1}^{n} x_{i j}^{2} / c_{i j}^{2}\right)^{2}\right]-m\left\{E\left[\hat{\Phi}_{r}\right][n(n-1)-2 m]+2 m\right\}+m^{2}\right)}{[n(n-1)-2 m]^{2}}-E^{2}\left[\hat{\Phi}_{r}\right] \\
= & \frac{4\left\{E\left[\left(\sum_{i=1}^{n} \sum_{j=1}^{n} x_{i j}^{2} / c_{i j}^{2}\right)^{2}\right]-m E\left[\hat{\Phi}_{r}\right][n(n-1)-2 m]-m^{2}\right\}}{[n(n-1)-2 m]^{2}}-E^{2}\left[\hat{\Phi}_{r}\right] .
\end{aligned}
$$


It thus only remains to solve the following term in order to obtain the expected variance for the estimator under a specific null hypothesis (see Appendix B):

$$
E\left[\left(\sum_{i=1}^{n} \sum_{j=1}^{n} x_{i j}^{2} / c_{i j}^{2}\right)^{2}\right] .
$$

Finally, its standard error $(S E)$ is equal to

$$
\begin{gathered}
\sigma\left(\hat{\Phi}_{r}\right)=\frac{2 \sqrt{\sum_{i=1}^{n} \sum_{j=i+1}^{n} \frac{q_{i j}+q_{j i}+2 s_{i j}}{c_{i j}^{4}}}}{n(n-1)-2 m}, \\
q_{i j}+q_{j i}+2 s_{i j}= \\
+c_{i j}\left(\pi_{i j}-7 \pi_{i j}^{2}+12 \pi_{i j}^{3}-6 \pi_{i j}^{4}\right) \\
+8 c_{i j}^{2}\left(-\pi_{i j}+6 \pi_{i j}^{2}-10 \pi_{i j}^{3}+5 \pi_{i j}^{4}\right) \\
+4 c_{i j}^{3}\left(\pi_{i j}-5 \pi_{i j}^{2}+8 \pi_{i j}^{3}-4 \pi_{i j}^{4}\right) .
\end{gathered}
$$

Note that the estimator is consistent, since its $S E$ approaches 0 as $n$ increases.

If $\pi_{i j}=.5$ for all $i$ and $j$, the $S E$ can be computed by means of the following formula:

$$
\sigma\left(\hat{\Phi}_{r}\right)=\frac{\sqrt{2 \sum_{i=1}^{n} \sum_{j=i+1}^{n} \frac{c_{i j}-1}{c_{i j}^{3}}}}{n(n-1)-2 m} .
$$

Social researchers may be interested in comparing $\hat{\Phi}_{r}$ with other measures of social reciprocity (e.g., directional consistency; van Hooff \& Wensing, 1987). This comparison can be carried out by means of the mean square error $\left(M S_{\mathrm{e}}\right)$, which is computed as follows for an estimator $\hat{\theta}$ :

$$
M S_{\mathrm{e}}(\hat{\theta})=E^{2}[\hat{\theta}-\theta]+\sigma^{2}(\hat{\theta})=\operatorname{Bias}^{2}(\hat{\theta})+\sigma^{2}(\hat{\theta}) .
$$

Then the $M S_{\mathrm{e}}$ for $\hat{\boldsymbol{\Phi}}_{r}$ equals

$$
\begin{aligned}
M S_{\mathrm{e}}\left(\hat{\Phi}_{r}\right) & =E^{2}\left[\hat{\Phi}_{r}-\Phi_{r}\right]+\sigma^{2}\left(\hat{\Phi}_{r}\right) \\
& =\operatorname{Bias}^{2}\left(\hat{\Phi}_{r}\right)+\sigma^{2}\left(\hat{\Phi}_{r}\right) .
\end{aligned}
$$

\section{The Null Hypothesis}

Social researchers are often interested in testing whether social relations among individuals in a group are symmetrical, which is why they often test dyadic and generalized reciprocity. In this regard, the null hypothesis for complete reciprocation can be expressed as follows:

$$
H_{0}: \Phi_{r}=0 .
$$

This statistical hypothesis is equivalent to $\pi_{i j}=\pi_{j i}=.5$ for all $i$ and $j$. Thus, if the null hypothesis is rejected, we can conclude that social interactions within a group are partially asymmetrical, at least with regard to the behavior of interest. In other words, individuals do not completely reciprocate each other's behavior. Note that rejecting the null hypothesis does not tell us anything about the degree of asymmetrical relations because we only have some evi- dence that $\hat{\boldsymbol{\Phi}}_{r}$ is different from zero. At all events, the values of $\hat{\Phi}_{r}$ are point estimates of the actual degree of asymmetry in groups, and researchers should take into account the estimator's bias when interpreting their results. On the other hand, if the null hypothesis is accepted, this would still not allow social researchers to conclude that individuals within a group completely reciprocate; statistical tests do not completely control Type II error.

Social relations within a group will be asymmetrical if $\pi_{i j} \neq \pi_{j i}$ for any $i$ and $j$; therefore, we should conclude that there is at least one dyad for which $\pi_{i j} \neq \pi_{j i}$ if the null hypothesis is rejected.

\section{Statistical Significance}

For any value of the test statistic, it is useful to know its exact statistical significance. In order to obtain exact statistical significance, it is necessary to establish all possible $c_{i j}$ values for a given $n$ and to compute the test statistic for all admissible $x_{i j}$ results. However, because there are so many possibilities, this task is not practical; this is a problem that has been encountered in other dyadic methods (Landau, 1951; Rapoport, 1949). To assess statistical significance, we propose employing Monte Carlo sampling. This statistical method becomes particularly useful if the population distribution is known but the sampling distribution of the estimator of interest has not been analytically derived (Noreen, 1989). Note that the sampling distribution of the test statistic can be estimated since all $x_{i j}$ are supposed to be binomially distributed.

A computer code has been developed for testing asymmetry in groups-specifically SAS/IML and R functions - and is available upon request. To carry out the simulation, the program needs the original matrix $\mathbf{X}$ to compute the values $c_{i j}$ from $x_{i j}$ and $x_{j i}$ and the number of individuals $n$. Additionally, the null hypothesis must be specified - that is, the values $\pi_{i j}$. The program then computes $\hat{\Phi}_{r}$, its mathematical expectancy and $S E$, and the dyadic and individual indexes.

Probabilities $\pi_{i j}=.5$ are used to obtain simulated sociomatrices if the complete reciprocation hypothesis is tested. For each dyad, the value $x_{i j}$ is drawn at random, and $x_{j i}$ is computed by subtracting $x_{i j}$ from $c_{i j}$. The simulated interaction matrix is then used to compute the test statistic. This process is iterated $N S$ times, where $N S$ denotes the number of simulated sociomatrices. The one-tailed statistical significance of the test equals $p=(N O S+1) /$ $(N S+1)$, where $N O S$ denotes the number of significant samples. That is, NOS is equal to the number of simulated sociomatrices for which the value of the test statistic is at least as large as the observed value in the original sociomatrix. This Monte Carlo procedure, which is included in the computer program, allows social researchers to obtain statistical significance values for the global, dyadic, and individual statistics under any null hypothesis, with regard to the degree of social reciprocity.

\section{An Example}

The following matrix concerns aggressive behavior in children and represents the number of aggressive acts that each child has addressed to others during play interaction 
Table 1

Individuals' Social Asymmetry Contributions As Actors and As Partners

\begin{tabular}{|c|c|c|c|c|c|c|c|}
\hline \multirow[b]{2}{*}{$\hat{\phi}_{i j}$} & \multicolumn{6}{|c|}{ Individual } & \multirow[b]{2}{*}{$\hat{\phi}_{i a}$} \\
\hline & 1 & 2 & 3 & 4 & 5 & 6 & \\
\hline 1 & 0 & $.004298^{\dagger}$ & $-.014586^{\dagger}$ & $.023900^{* *}$ & $-.020888^{*}$ & $.032006^{\dagger}$ & $.024730^{\dagger}$ \\
\hline 2 & $-.004037^{\dagger}$ & 0 & $-.032211^{* *}$ & $.034698^{* *}$ & $.030577^{*}$ & $.013927^{* *}$ & $.042954^{*}$ \\
\hline 3 & $.018753^{\dagger}$ & $.076526^{* *}$ & 0 & $.023771^{* *}$ & $.057603^{* *}$ & $.061398^{* *}$ & $.238051^{* *}$ \\
\hline 4 & $-.017487^{* *}$ & $-.022455^{* *}$ & $-.017412^{* *}$ & 0 & $-.014866^{* *}$ & $.002642^{\dagger}$ & $-.069578^{* *}$ \\
\hline 5 & $.030972^{*}$ & $-.020714^{*}$ & $-.029369^{* *}$ & $.019221^{* *}$ & 0 & $.005154^{\dagger}$ & $.005264^{\dagger}$ \\
\hline 6 & $-.021337^{\dagger}$ & $-.011504^{* *}$ & $-.030046^{* *}$ & $-.002541^{\dagger}$ & $-.004798^{\dagger}$ & 0 & $-.070226^{* *}$ \\
\hline$\hat{\phi}_{p j}$ & $.006864^{\dagger}$ & $.026151^{\dagger}$ & $-.123624^{* *}$ & $.099049^{* *}$ & $.047628^{*}$ & $.115127^{* *}$ & .1712 \\
\hline
\end{tabular}

Note- $\hat{\phi}_{i a}$, actor's social asymmetry contribution; $\hat{\phi}_{p j}$, partner's social asymmetry contribution; $\hat{\phi}_{i j}$, contribution associated with each partner. $p$ values were estimated by means of a Monte Carlo procedure with 99,999 simulated matrices under the null hypothesis of complete reciprocation. ${ }^{\dagger}$.. . . ${ }^{*} p<.05 .{ }^{* *} p<.01$.

(Kenny et al., 2007; printed with permission), with rows and columns representing individuals as actors and partners, respectively:

$$
\mathbf{X}=\left(\begin{array}{cccccc}
0 & 17 & 12 & 57 & 11 & 14 \\
15 & 0 & 6 & 95 & 18 & 128 \\
20 & 59 & 0 & 89 & 19 & 59 \\
30 & 38 & 47 & 0 & 83 & 294 \\
25 & 8 & 4 & 140 & 0 & 36 \\
6 & 87 & 11 & 272 & 31 & 0
\end{array}\right) .
$$

The value of the statistic for the matrix $\mathbf{X}$ is approximately equal to .1712 , and the expected value for the estimator equals .0201 under the null hypothesis of complete reciprocation. This shows that the empirical value is clearly larger than the expected value. With regard to the variance and the $S E$ of the estimator, these are equal, respectively, to .000082 and .009038 under the null hypothesis of social reciprocity in the group. Note that the difference between the statistic's value and the expected value is large enough in comparison with the $S E$ for suggesting that the individuals of the group are nonreciprocal at the global level. As an initial descriptive analysis, these results suggest that the individuals of the group are nonreciprocal at the global level, with regard to aggressive behavior.

Although descriptive analysis is illustrative, a statistical decision regarding the null hypothesis that establishes the complete reciprocation between individuals is also needed. In order to make a statistical decision with respect to the null hypothesis of complete reciprocation, the statistical significance of the value of the asymmetry statistic was estimated using a Monte Carlo simulation, in which we ran 99,999 iterations. The obtained $p$ value was less than .0001 , which clearly suggests that the null hypothesis is unlikely. As a conclusion, at least one pair of children was responsible for the asymmetry in these dyadic relations. That is, there was at least one child in the group who did not reciprocate in aggression.

We know that the lack of social reciprocity in the group at the individual level was mainly explained by Individual 3 as an actor; but, each as a partner, Individuals 3 and 6 accounted for the main part of asymmetry (see Table 1). That is, Individual 3 was the child who addressed the most aggression and received less aggression from others (positive value of $\hat{\phi}_{i a}$ and negative value of $\hat{\phi}_{p j}$, respectively). Additionally, Individual 6 received more aggressive behavior from partners than she or he addressed to them.

At the dyadic level, Dyad 2-3 made the largest contribution to asymmetry (see Table 2). However, all of the dyads showed very low values of contribution to asymmetry. When a Monte Carlo procedure was carried out for testing the null hypothesis of complete reciprocation, 6 of the 15 dyads in the group - specifically, Dyads $1-2$, $1-3,1-6,2-5,4-6$, and 5-6 - appeared not to have made significant contributions to asymmetry in the dyadic relationship $\left(\hat{\Phi}_{i j}\right)$. These results were confirmed when a Monte Carlo sampling was carried out for the directional dyadic contributions to asymmetry (see $\hat{\phi}_{i j}$ measures in Table 1$)$, with the exception of Dyad 2-5. Thus, Individual 1's asymmetry addressed toward Individuals 2, 3, and 6 (and

Table 2

Contributions to Overall Asymmetry by the Dyad $i-j$

\begin{tabular}{ccccccc}
\hline & \multicolumn{6}{c}{ Individual } \\
\cline { 2 - 7 }$\hat{\Phi}_{i j}$ & 1 & 2 & 3 & 4 & 5 & 6 \\
\hline 1 & 0 & $.00026^{\dagger}$ & $.004167^{\dagger}$ & $.006413^{* *}$ & $.010084^{*}$ & $.010669^{\dagger}$ \\
2 & .00026 & 0 & $.044315^{* *}$ & $.012243^{* *}$ & $.009864^{\dagger}$ & $.002423^{* *}$ \\
3 & .004167 & .044315 & 0 & $.006359^{* *}$ & $.028234^{* *}$ & $.031352^{* *}$ \\
4 & .006413 & .012243 & .006359 & 0 & $.004355^{* *}$ & $.000101^{\dagger}$ \\
5 & .010084 & .009864 & .028234 & .004355 & 0 & $.000356^{\dagger}$ \\
6 & .010669 & .002423 & .031352 & .000101 & .000356 & 0 \\
\hline
\end{tabular}

Note- $\hat{\Phi}_{i j}$, overall asymmetry contributions by Dyad $i-j . p$ values were estimated by means of a Monte Carlo procedure with 99,999 simulated matrices under the null hypothesis of complete reciprocation. in.s. ${ }^{*} p<.05 .{ }^{* *} p<.01$. 
vice versa) and Individual 6's asymmetry addressed to Individual 5 (and vice versa) were not significantly different from what was expected under the null hypothesis, which predicted complete reciprocation in aggressive behavior. In contrast, the directional dyadic contributions to the lack of balance in aggression for Individuals 2 and 5 , both $\hat{\phi}_{25}$ and $\hat{\phi}_{52}$, were significant at the $5 \%$ level.

\section{Conclusions}

The findings of the present research are intended for use by researchers interested in measuring and testing asymmetric relationships in groups. The proposed statistic is founded on values $x_{i j}$ of sociomatrices $\mathbf{X}$. This statistic enables social researchers to assess social asymmetry because discrepancies between what each individual gives others and what is received in return is taken into account. Note that the product-moment correlation coefficient remains unchanged under location and scale transformations. This characteristic of the correlation coefficient is adequate if social researchers are interested in estimating generalized reciprocity, but it does not lead them to a precise assessment of absolute dyadic reciprocity; the product-moment correlation coefficient does not take into account differences between what individuals give others and what they receive from them in return.

The proposed statistic $\hat{\Phi}_{r}$ is bounded by 0 and 1 , which refer, respectively, to the maximum and minimum of social reciprocity. Thus, if the statistic is equal to 0 , there will be a complete reciprocation in groups, whereas a value close to 1 denotes asymmetric relationships (i.e., the larger the value of the index, the lower the dyadic reciprocity among individuals). Hence, the statistic allows social researchers to measure the degree of social reciprocity at the group level. Additionally, the statistic has been decomposed into components to determine the contribution of each individual and of each dyad to asymmetry.

Regarding statistical inference, we have demonstrated that the estimator $\hat{\Phi}_{r}$ is biased and that this kind of statistical error should be considered when one is interpreting estimated values of social reciprocity in groups. Since mathematical expectancy has been analytically derived for this estimator, social researchers are able to determine whether the test statistic takes a value larger than the expected value under the null hypothesis. In other words, researchers should not expect the test statistic to be equal to 0 in samples if there is actually complete reciprocation in populations.

We have also analytically derived the $S E$ for the sampling distribution of the estimator. Since the bias and the $S E$ of the estimator are known, it is feasible to compare this test statistic with others. Thus, the mean square error can be computed and used to select the best estimator. Unfortunately, since the sampling distribution of the estimator is not known, we cannot propose an exact statistical test. Further work is needed to determine the exact sampling distribution for the estimator. Since the exact sampling distribution of the estimator has not been derived, we proposed using the Monte Carlo method to estimate statistical significance. Briefly, the method consists of randomly sampling sociomatrices from a population whose parameters are in accordance with the null hypothesis and where the number of individuals is equal to that of the original sample. All test statistic values of simulated samples are arranged in ascending order, and the original value is located in this distribution. In other words, the algorithm computes the number of simulated test statistic values that are at least as large as the test statistic for the original sociomatrix. This estimate of statistical significance enables social researchers to make statistical decisions with regard to the null hypothesis. We suggest specifying a large number of simulated sociomatrices in the algorithm in order to improve statistical significance estimates. Note that the reliability of these statistical significance estimates increases with the number of simulated sociomatrices.

From an applied point of view, we can envision several applications of the social reciprocity statistic for a variety of topics in social psychology and social ethology - for instance, interpersonal perception (Kenny, 1994), social conflict and reconciliation in gregarious species (Cooper, Bernstein, \& Hemelrijk, 2005), and agonistic dominance (Vervaecke, de Vries, \& van Elsacker, 1999).

In summary, the present research describes a new procedure for measuring and making statistical decisions with regard to social reciprocity at global, dyadic, and individual levels. Whereas other indexes and statistics are based on association coefficients or do not enable social researchers to obtain dyadic and individual effects, the proposed statistical method allows researchers not only to quantify the lack of social reciprocity as a function between what is given to others and what is received in return, but also to estimate dyadic and individual effects. Additionally, a Monte Carlo method is proposed for obtaining approximate statistical significance. We highlight that the estimator's values can also be obtained for sociomatrices with incomplete data.

\section{AUTHOR NOTE}

This research was partially supported by Ministerio de Educación y Ciencia Grants SEJ2005-07310-C02-01/PSIC and SEJ2005 -07310-C02-02/PSIC, by the Comissionat per a Universitats i Recerca of the Departament d'Innovació, by the Universitats i Empresa of the Generalitat de Catalunya, and by European Social Fund Grants 2005SGR00098 and 2008FIC00156. We thank Antonius Cillessen for allowing us partial use of data that he had recorded for a previous study. Correspondence concerning this article should be addressed to A. Solanas, Departament de Metodologia de les Ciències del Comportament, Facultat de Psicologia, Universitat de Barcelona, Passeig de la Vall d'Hebron, 171, 08035-Barcelona, Spain (e-mail: antonio.solanas@ ub.edu).

\section{REFERENCES}

Bond, C. F., JR., Horn, E. M., \& Kenny, D. A. (1997). A model for triadic relations. Psychological Methods, 2, 79-94.

Campbell, L., \& Kashy, D. A. (2002). Estimating, actor, partner, and interaction effects for dyadic data using PROC MIXED and HLM: A user-friendly guide. Personal Relationships, 9, 327-342.

Cooper, M. A., Bernstein, I. S., \& HemelriJK, C. K. (2005). Reconciliation and relationship quality in assamese macaques (Macaca assamensis). American Journal of Primatology, 65, 269-282.

DE VRIES, H. (1995). An improved test of linearity in dominance hierarchies containing unknown or tied relationships. Animal Behaviour, 50, 1375-1389. 
DE VRIES, H. (1998). Finding a dominance order most consistent with a linear hierarchy: A new procedure and review. Animal Behaviour, $\mathbf{5 5}, 827-843$.

DE VRIES, H., \& Appleby, M. C. (2000). Finding an appropriate order for a hierarchy: A comparison of the I\&SI and the BBS methods. Animal Behaviour, 59, 239-245.

de Vries, H., Stevens, J. M. G., \& Vervaecke, H. (2006). Measuring and testing the steepness of dominance hierarchies. Animal Behaviour, 71, 585-592.

GonZalez, R., \& Griffin, D. (1999). The correlational analysis of dyad-level data in the distinguishable case. Personal Relationships, 6, 449-469.

GrifFin, D., \& Gonzalez, R. (1995). Correlational analysis of dyad-level data in the exchangeable case. Psychological Bulletin, 118, 430-439.

HemelRIJK, C. K. (1990a). A matrix partial correlation test used in investigations of reciprocity and other social interaction patterns at a group level. Journal of Theoretical Biology, 143, 405-420.

HeMELRIJK, C. K. (1990b). Models of, and tests for, reciprocity, unidirectionality and other social interaction patterns at a group level. Animal Behaviour, 39, 1013-1029.

Johnson, N. L., Kotz, S., \& Kemp, A. W. (1992). Univariate discrete distributions (2nd ed.). New York: Wiley.

Kendall, M. G., \& Babington Smith, B. (1940). On the method of paired comparisons. Biometrika, 31, 324-345.

KENNY, D. A. (1994). Interpersonal perception: A social relations analysis. New York: Guilford.

Kenny, D. A., Kashy, D. A., \& CoOK, W. L. (2006). Dyadic data analysis. New York: Guilford.

Kenny, D. A., \& LA VoIE, L. (1984). The social relations model. In L. Berkowitz (Ed.), Advances in experimental psychology (Vol. 18, pp. 142-182). San Diego: Academic Press.
Kenny, D. A., West, T. V., Cillessen, A. H. N., Coie, J. D., Dodge, K. A., Hubbard, J. A., \& Schwartz, D. (2007). Accuracy in judgments of aggressiveness. Personality \& Social Psychology Bulletin, 33, 1225-1236.

LANDAU, H. G. (1951). On dominance relations and the structure of animal societies: I. Effect of inherent characteristics. Bulletin of Mathematical Biophysics, 13, 1-19.

Leiva, D., Solanas, A., \& Salafranca, L. (2008). Testing reciprocity in social interactions: A comparison between the directional consistency and skew-symmetry statistics. Behavior Research Methods, 40, 626-634.

NoReEn, E. W. (1989). Computer-intensive methods for testing hypotheses: An introduction. New York: Wiley.

RAPOPORT, A. (1949). Outline of a probabilistic approach to animal sociology: I. Bulletin of Mathematical Biophysics, 11, 183-196.

SAITO, T., \& YADOHISA, H. (2005). Data analysis of asymmetric structures: Advanced approaches in computational statistics. New York: Dekker.

Solanas, A., Salafranca, L., Riba, C., Sierra, V., \& Leiva, D. (2006). Quantifying social asymmetric structures. Behavior Research Methods, 38, 390-399.

van Hooff, J. A. R. A. M., \& Wensing, J. A. B. (1987). Dominance and its behavioural measures in a captive wolf pack. In H. W. Frank (Ed.), Man and wolf (pp. 219-252). Dordrecht, The Netherlands: Junk Publishers.

Vervaecke, H., de Vries, H., \& van Elsacker, L. (1999). An experimental evaluation of the consistency of competitive ability and agonistic dominance in different social contexts in captive bonobos. Behaviour, 136, 423-442.

Warner, R. M., Kenny, D. A., \& Stoto, M. (1979). A new round robin analysis of variance for social interaction data. Journal of Personality \& Social Psychology, 37, 1742-1757.

\section{The Mathematical Expectancy of the Estimator Under a Specific Null Hypothesis}

The expected value for $\hat{\Phi}_{r}$ is equal to

$$
E\left[\hat{\Phi}_{r}\right]=E\left[\frac{2 \sum_{\substack{i=1 \\ j}}^{n} \sum_{j=1}^{n} x_{i j}^{2} / c_{i j}^{2}-2 m}{n(n-1)-2 m}\right]=\frac{2 \sum_{\substack{i=1 \\ j \neq 1 \\ j \neq i}}^{n} E\left[x_{i j}^{2}\right] / c_{i j}^{2}-2 m}{n(n-1)-2 m} .
$$

If $x_{i j}$ follows a binomial distribution, $E\left[x_{i j}^{2}\right]$, which is the second moment about zero, can be written as follows (Johnson, Kotz, \& Kemp, 1992):

$$
E\left[x_{i j}^{2}\right]=c_{i j} \pi_{i j}+c_{i j}\left(c_{i j}-1\right) \pi_{i j}^{2} .
$$

Thus,

$$
\begin{gathered}
E\left[\hat{\Phi}_{r}\right]=\frac{2 \sum_{i=1}^{n} \sum_{\substack{j=1 \\
j \neq i}}^{n}\left[c_{i j} \pi_{i j}+c_{i j}\left(c_{i j}-1\right) \pi_{i j}^{2}\right] / c_{i j}^{2}-2 m}{n(n-1)-2 m}=\frac{2 \sum_{i=1}^{n} \sum_{\substack{j=1 \\
j \neq i}}^{n}\left(c_{i j} \pi_{i j}+c_{i j}^{2} \pi_{i j}^{2}-c_{i j} \pi_{i j}^{2}\right) / c_{i j}^{2}-2 m}{n(n-1)-2 m} \\
=\frac{2\left[\sum_{i=1}^{n} \sum_{j=i+1}^{n} 1 / c_{i j}+\sum_{i=1}^{n} \sum_{\substack{j=1 \\
j \neq i}}^{n} \pi_{i j}^{2}-\sum_{i=1}^{n} \sum_{\substack{j=1 \\
j \neq i}}^{n} \pi_{i j}^{2} / c_{i j}-m\right)}{n(n-1)-2 m} \\
=\frac{2\left[\sum_{i=1}^{n} \sum_{j=i+1}^{n} 1 / c_{i j}+\sum_{i=1}^{n} \sum_{\substack{j=1 \\
j \neq i}}^{n} \frac{\pi_{i j}^{2}\left(c_{i j}-1\right)}{c_{i j}}-m\right]}{n(n-1)-2 m} .
\end{gathered}
$$




\section{APPENDIX A (Continued)}

If there are dyads for which $c_{i j}$ equals 0 , mathematical expectancy can be obtained as follows:

$$
E\left[\hat{\Phi}_{r}\right]=\frac{2\left[\sum_{\substack { i=1 \\
\begin{subarray}{c}{j=i+1 \\
c_{i j} \neq 0{ i = 1 \\
\begin{subarray} { c } { j = i + 1 \\
c _ { i j } \neq 0 } }\end{subarray}}^{n} 1 / c_{i j}+\sum_{\substack { i=1 \\
\begin{subarray}{c}{j=1 \\
j \neq i \\
c_{i j} \neq 0{ i = 1 \\
\begin{subarray} { c } { j = 1 \\
j \neq i \\
c _ { i j } \neq 0 } }\end{subarray}}^{n} \frac{\pi_{i j}^{2}\left(c_{i j}-1\right)}{c_{i j}}-m\right]}{2 z-2 m} .
$$

\section{APPENDIX B}

The variance of the estimator for a specific null hypothesis equals

$$
\begin{aligned}
\sigma^{2}\left(\hat{\Phi}_{r}\right)= & \frac{4\left(E\left[\left(\sum_{i=1}^{n} \sum_{j=1}^{n} x_{i j}^{2} / c_{i j}^{2}\right)^{2}\right]-m\left\{E\left[\hat{\Phi}_{r}\right][n(n-1)-2 m]+2 m\right\}+m^{2}\right)}{[n(n-1)-2 m]^{2}}-E^{2}\left[\hat{\Phi}_{r}\right] \\
= & \frac{4\left(E\left[\left(\sum_{i=1}^{n} \sum_{j=1}^{n} x_{i j}^{2} / c_{i j}^{2}\right)^{2}\right]-m E\left[\hat{\Phi}_{r}\right][n(n-1)-2 m]-m^{2}\right)}{[n(n-1)-2 m]^{2}}-E^{2}\left[\hat{\Phi}_{r}\right] .
\end{aligned}
$$

Note that

$$
\begin{aligned}
E\left[\left(\sum_{\substack{i=1 \\
j \neq 1 \\
j \neq i}}^{n} \sum_{i j}^{n} x_{i j}^{2} / c_{i j}^{2}\right]\right. & =E^{2}\left(\sum_{\substack{i=1 \\
j \neq 1 \\
j \neq i}}^{n} \sum_{i j}^{n} x_{i j}^{2} / c_{i j}^{2}\right)+\operatorname{Var}\left(\sum_{\substack{i=1 \\
j \neq 1 \\
j \neq i}}^{n} \sum_{i j}^{n} / c_{i j}^{2}\right) \\
& =E^{2}\left(\sum_{\substack{i=1 \\
j}}^{n} \sum_{\substack{j=1 \\
j \neq i}}^{n} x_{i j}^{2} / c_{i j}^{2}\right)+\sum_{\substack{i=1 \\
j}}^{n} \sum_{\substack{j \neq 1 \\
j \neq i}}^{n} \operatorname{Var}\left(x_{i j}^{2}\right) / c_{i j}^{4}+2 \sum_{i=1}^{n} \sum_{j=i+1}^{n} \operatorname{Cov}\left(x_{i j}^{2} / c_{i j}^{2}, x_{j i}^{2} / c_{i j}^{2}\right),
\end{aligned}
$$

where

$$
E^{2}\left[\sum_{\substack{i=1 \\ j}}^{n} \sum_{\substack{j=1 \\ j \neq i}}^{n} x_{i j}^{2} / c_{i j}^{2}\right]=\left\{\frac{E\left[\hat{\Phi}_{r}\right][n(n-1)-2 m]+2 m}{2}\right\}^{2} .
$$

Now, the variance for $x_{i j}^{2}$ has to be calculated. Thus,

$$
\begin{aligned}
\operatorname{Var}\left(x_{i j}^{2}\right)=E\left[x_{i j}^{4}\right]-E^{2}\left[x_{i j}^{2}\right]= & c_{i j} \pi_{i j}+7 c_{i j}\left(c_{i j}-1\right) \pi_{i j}^{2}+6 c_{i j}\left(c_{i j}-1\right)\left(c_{i j}-2\right) \pi_{i j}^{3} \\
& +c_{i j}\left(c_{i j}-1\right)\left(c_{i j}-2\right)\left(c_{i j}-3\right) \pi_{i j}^{4}-\left[c_{i j} \pi_{i j}+\left(c_{i j}^{2}-c_{i j}\right) \pi_{i j}^{2}\right]^{2}
\end{aligned}
$$

since the fourth moment about zero for a binomially distributed variable is equal to (see Johnson et al., 1992)

$$
E\left[x_{i j}^{4}\right]=c_{i j} \pi_{i j}+7 c_{i j}\left(c_{i j}-1\right) \pi_{i j}^{2}+6 c_{i j}\left(c_{i j}-1\right)\left(c_{i j}-2\right) \pi_{i j}^{3}+c_{i j}\left(c_{i j}-1\right)\left(c_{i j}-2\right)\left(c_{i j}-3\right) \pi_{i j}^{4} .
$$

After some algebraic operations, we obtain

$$
\begin{aligned}
\operatorname{Var}\left(x_{i j}^{2}\right)= & c_{i j} \pi_{i j}+7\left(c_{i j}^{2}-c_{i j}\right) \pi_{i j}^{2}+6\left(c_{i j}^{3}-3 c_{i j}^{2}+2 c_{i j}\right) \pi_{i j}^{3}+\left(c_{i j}^{4}-6 c_{i j}^{3}+11 c_{i j}^{2}-6 c_{i j}\right) \pi_{i j}^{4} \\
& -c_{i j}^{2} \pi_{i j}^{2}+2 c_{i j}^{2} \pi_{i j}^{3}-2 c_{i j}^{3} \pi_{i j}^{3}-c_{i j}^{2} \pi_{i j}^{4}+2 c_{i j}^{3} \pi_{i j}^{4}-c_{i j}^{4} \pi_{i j}^{4} \\
= & c_{i j} \pi_{i j}-7 c_{i j} \pi_{i j}^{2}+6 c_{i j}^{2} \pi_{i j}^{2}+12 c_{i j} \pi_{i j}^{3}-16 c_{i j}^{2} \pi_{i j}^{3}+4 c_{i j}^{3} \pi_{i j}^{3}-6 c_{i j} \pi_{i j}^{4}+10 c_{i j}^{2} \pi_{i j}^{4}-4 c_{i j}^{3} \pi_{i j}^{4} \\
= & c_{i j}\left(\pi_{i j}-7 \pi_{i j}^{2}+12 \pi_{i j}^{3}-6 \pi_{i j}^{4}\right)+c_{i j}^{2}\left(6 \pi_{i j}^{2}-16 \pi_{i j}^{3}+10 \pi_{i j}^{4}\right)+c_{i j}^{3}\left(4 \pi_{i j}^{3}-4 \pi_{i j}^{4}\right)=q_{i j} .
\end{aligned}
$$

It can also be shown that 


\section{APPENDIX B (Continued)}

$$
\begin{aligned}
\operatorname{Var}\left(x_{j i}^{2}\right)= & c_{i j}\left(\pi_{i j}-7 \pi_{i j}^{2}+12 \pi_{i j}^{3}-6 \pi_{i j}^{4}\right)+c_{i j}^{2}\left(-4 \pi_{i j}+18 \pi_{i j}^{2}-24 \pi_{i j}^{3}+10 \pi_{i j}^{4}\right) \\
& +c_{i j}^{3}\left(4 \pi_{i j}-12 \pi_{i j}^{2}+12 \pi_{i j}^{3}-4 \pi_{i j}^{4}\right)=q_{j i} .
\end{aligned}
$$

Thus,

$$
\begin{aligned}
q_{i j}+q_{j i}= & 2 c_{i j}\left(\pi_{i j}-7 \pi_{i j}^{2}+12 \pi_{i j}^{3}-6 \pi_{i j}^{4}\right)+c_{i j}^{2}\left(-4 \pi_{i j}+24 \pi_{i j}^{2}-40 \pi_{i j}^{3}+20 \pi_{i j}^{4}\right) \\
& +c_{i j}^{3}\left(4 \pi_{i j}-12 \pi_{i j}^{2}+16 \pi_{i j}^{3}-8 \pi_{i j}^{4}\right),
\end{aligned}
$$

and we can write

$$
\sum_{i=1}^{n} \sum_{\substack{j=1 \\ j \neq i}}^{n} \frac{\operatorname{Var}\left(x_{i j}^{2}\right)}{c_{i j}^{4}}=\sum_{i=1}^{n} \sum_{j=i+1}^{n} \frac{q_{i j}+q_{j i}}{c_{i j}^{4}} .
$$

Now we will solve the covariances,

$$
\begin{aligned}
\sum_{i=1}^{n} \sum_{j=i+1}^{n} \operatorname{Cov}\left(x_{i j}^{2} / c_{i j}^{2}, x_{j i}^{2} / c_{i j}^{2}\right) & =\sum_{i=1}^{n} \sum_{j=i+1}^{n} \frac{\operatorname{Cov}\left(x_{i j}^{2}, x_{j i}^{2}\right)}{c_{i j}^{4}} \\
& =\sum_{i=1}^{n} \sum_{j=i+1}^{n} \frac{E\left[\left(x_{i j}^{2}-E\left[x_{i j}^{2}\right]\right)\left(x_{j i}^{2}-E\left[x_{j i}^{2}\right]\right)\right]}{c_{i j}^{4}} \\
& =\sum_{i=1}^{n} \sum_{j=i+1}^{n} \frac{E\left[x_{i j}^{2} x_{j i}^{2}\right]-E\left[x_{i j}^{2}\right] E\left[x_{j i}^{2}\right]}{c_{i j}^{4}},
\end{aligned}
$$

given that

$$
\begin{aligned}
& E\left[x_{i j}^{2} x_{j i}^{2}\right]=E\left[x_{i j}^{2}\left(c_{i j}-x_{i j}\right)^{2}\right]=c_{i j}^{2} E\left[x_{i j}^{2}\right]-2 c_{i j} E\left[x_{i j}^{3}\right]+E\left[x_{i j}^{4}\right], \\
& E\left[x_{i j}^{2}\right] E\left[x_{j i}^{2}\right]=E\left[x_{i j}^{2}\right] E\left[\left(c_{i j}-x_{i j}\right)^{2}\right]=c_{i j}^{2} E\left[x_{i j}^{2}\right]-2 c_{i j} E\left[x_{i j}\right] E\left[x_{i j}^{2}\right]+E^{2}\left[x_{i j}^{2}\right] .
\end{aligned}
$$

Then,

$$
\begin{aligned}
\operatorname{Cov}\left(x_{i j}^{2}, x_{j i}^{2}\right)= & E\left[x_{i j}^{4}\right]-2 c_{i j} E\left[x_{i j}^{3}\right]+2 c_{i j} E\left[x_{i j}\right] E\left[x_{i j}^{2}\right]-E^{2}\left[x_{i j}^{2}\right] \\
= & c_{i j}\left(\pi_{i j}-7 \pi_{i j}^{2}+12 \pi_{i j}^{3}-6 \pi_{i j}^{4}\right)+c_{i j}^{2}\left(-2 \pi_{i j}+12 \pi_{i j}^{2}-20 \pi_{i j}^{3}+10 \pi_{i j}^{4}\right) \\
& +c_{i j}^{3}\left(-4 \pi_{i j}^{2}+8 \pi_{i j}^{3}-4 \pi_{i j}^{4}\right)=s_{i j}=s_{j i},
\end{aligned}
$$

since the third moment about zero for a binomially distributed variable is equal to (see Johnson et al., 1992)

$$
E\left[x_{i j}^{3}\right]=c_{i j} \pi_{i j}+3 c_{i j}\left(c_{i j}-1\right) \pi_{i j}^{2}+c_{i j}\left(c_{i j}-1\right)\left(c_{i j}-2\right) \pi_{i j}^{3} .
$$

It can be shown that

$$
\begin{aligned}
q_{i j}+q_{j i}+2 s_{i j}= & 4 c_{i j}\left(\pi_{i j}-7 \pi_{i j}^{2}+12 \pi_{i j}^{3}-6 \pi_{i j}^{4}\right)+8 c_{i j}^{2}\left(-\pi_{i j}+6 \pi_{i j}^{2}-10 \pi_{i j}^{3}+5 \pi_{i j}^{4}\right) \\
& +4 c_{i j}^{3}\left(\pi_{i j}-5 \pi_{i j}^{2}+8 \pi_{i j}^{3}-4 \pi_{i j}^{4}\right) .
\end{aligned}
$$

Now we can write,

$$
\begin{aligned}
E\left[\left(\sum_{i=1}^{n} \sum_{\substack{j=1 \\
j \neq i}}^{n} x_{i j}^{2} / c_{i j}^{2}\right)^{2}\right] & =\left\{\frac{E\left[\hat{\Phi}_{r}\right][n(n-1)-2 m]+2 m}{2}\right\}^{2}+\sum_{i=1}^{n} \sum_{j=i+1}^{n} \frac{q_{i j}+q_{j i}}{c_{i j}^{4}}+2 \sum_{i=1}^{n} \sum_{j=i+1}^{n} \frac{s_{i j}}{c_{i j}^{4}} \\
& =\left\{\frac{E\left[\hat{\Phi}_{r}\right][n(n-1)-2 m]+2 m}{2}\right\}^{2}+\sum_{i=1}^{n} \sum_{j=i+1}^{n} \frac{q_{i j}+q_{j i}+2 s_{i j}}{c_{i j}^{4}}
\end{aligned}
$$

and, finally, 


\section{APPENDIX B (Continued)}

$$
\begin{aligned}
\sigma^{2}\left(\hat{\Phi}_{r}\right)= & \frac{\left\{E\left[\hat{\Phi}_{r}\right][n(n-1)-2 m]+2 m\right\}^{2}}{[n(n-1)-2 m]^{2}} \\
& +\frac{4\left\{\sum_{i=1}^{n} \sum_{j=i+1}^{n} \frac{q_{i j}+q_{j i}+2 s_{i j}}{c_{i j}^{4}}-m E\left[\hat{\Phi}_{r}\right][n(n-1)-2 m]-m^{2}\right\}}{[n(n-1)-2 m]^{2}}-E^{2}\left[\hat{\Phi}_{r}\right] \\
= & \frac{4 m E\left[\hat{\Phi}_{r}\right][n(n-1)-2 m]+4 m^{2}}{[n(n-1)-2 m]^{2}} \\
& +\frac{4\left\{\sum_{i=1}^{n} \sum_{j=i+1}^{n} \frac{q_{i j}+q_{j i}+2 s_{i j}}{c_{i j}^{4}}-m E\left[\hat{\Phi}_{r}\right][n(n-1)-2 m]-m^{2}\right\}}{[n(n-1)-2 m]^{2}} \\
= & \frac{4 \sum_{i=1}^{n} \sum_{j=i+1}^{n} \frac{q_{i j}+q_{j i}+2 s_{i j}}{c_{i j}^{4}}}{[n(n-1)-2 m]^{2}} .
\end{aligned}
$$

Again, when there are dyads for which $c_{i j}=0$, the variance can be obtained as follows:

$$
\sigma^{2}\left(\hat{\Phi}_{r}\right)=\frac{4 \sum_{i=1}^{n} \sum_{\substack{j=i+1 \\ c_{i j} \neq 0}}^{n} \frac{q_{i j}+q_{j i}+2 s_{i j}}{c_{i j}^{4}}}{(2 z-2 m)^{2}} .
$$

(Manuscript received September 29, 2008; accepted for publication December 16, 2008.) 